VII JORNADAS DE DIFUSIÓN DE LA INVESTIGACIÓN Y EXTENSIÓN - FCV-UNL

RESUMEN EXTENDIDO

\title{
DESARROLLO DE MODELO MURINO DE COLONIZACIÓN DE Lactobacillus plantarum LP5 DE ORIGEN PORCINO
}

\author{
Ruiz $\mathrm{MJ}^{1}$, Panzani $\mathrm{C}^{3}$, Fusari $\mathrm{M}^{2}$, Olivero $\mathrm{CR}^{1}$, Saluzzo $\mathrm{M}^{1}$, Frizzo $\mathrm{LS}^{12}$. \\ ${ }^{1}$ Laboratorio Análisis de Alimentos, ICIVET-Litoral, UNL-CONICET. \\ ${ }^{2}$ Departamento de Salud Pública, Facultad de Ciencias Veterinarias, UNL. \\ ${ }^{3}$ Centro de Medicina Comparada, ICIVET-Litoral, UNL-CONICET. \\ * Correspondencia: Ruiz MJ.E-mail: jruiz@vet.unicen.edu.ar \\ Editado por: R. Sobrero, V. Matiller, C. Baravalle.
}

DEVELOPMENT OF A MURINE COLONIZATION MODEL OF Lactobacillus plantarum LP5 OF PORCINE ORIGIN.

SUMMARY.

The objective of this work was to generate an experimental model of colonization by L. plantarum LP5 in mice (Mus musculus) aimed at improving the general state of the animal and preventing the possible presence of zoonotic pathogens. The mice used were females of the Balb/cCmedc strain, 6 weeks old. Animals were organized into 2 groups of 3 individuals: treated (GT) and control (GC). GT was administered twice weekly by gavage with $100 \mu \mathrm{l}$ of rifampicin resistant porcine L. plantarum LP5 at a concentration of 1010 CFU. Mice were fed water and food ad libitum throughout the experiment. Colonization in both groups was evaluated by the presence of clinical signs and by a microbiological analysis of the feces. Sampling was performed at weekly intervals to quantify the populations of the supplied L. plantarum LP5, Lactobacillus spp., Escherichia coli, total coliforms, Campylobacter and yeast. The results of the sampling in the GT in the week showed a recovery of L. plantarum LP5 above $5 \operatorname{logUFC/g}$, in the second it was 7 logUFC/g and in the third it was 5 $\operatorname{logUFC/g}$. The CG, meanwhile, maintained the null count throughout the experiment. The results showed that there was no imbalance between the microbial components. Therefore, it is feasible to affirm that the inoculum did not alter the balance of the intestinal ecosystem. Particularly, it did not exert an inhibitory effect on the members of the previously established microbiota.

Palabras clave: Modelo murino, Colonización, Lactobacillus plantarum, Bacteria ácido láctica.

Keywords: Murine model, Colonization, Lactobacillus plantarum, Lactic acid bacteria.

La utilización de bacterias ácido lácticas (BAL) como suplemento probiótico responde a una tendencia mundial que promueve una alimentación preventiva, sana, natural, de mayor calidad nutritiva y libre de residuos. Así se ha estimulado el interés por el uso de aditivos alimentarios y terapias alternativas no medicamentosas en reemplazo de los antibióticos utilizados en producción y sanidad animal, entre los cuales cabe destacar a los probióticos y las sustancias antimicrobianas naturales que ellos producen (Rosmini et al., 2004; Signorini et al., 2012). Se ha demostrado que algunas bacterias ácido lácticas (BAL) modifican, los patrones del flujo de nutrientes en el intestino, provocando efectos benéficos sobre la utilización del alimento por parte de los animales. A esto se suma la capacidad de inhibir patógenos intestinales in vitro y de proteger in vivo a animales infectados con bacterias patógenas (Vélez Zea et al., 2013). Por lo tanto, el uso 
de los microorganismos autóctonos bajo condición GRAS (Generally Recognised As Safe) con capacidad probiótica representa una interesante herramienta profiláctica para el tratamiento y prevención de enfermedades animales (Signorini et al., 2012). Lactobacillus plantarum es una BAL que presenta gran versatilidad de adaptación a nichos ambientales debido a la capacidad de codificación para la absorción y utilización de diferentes azúcares, captación de péptidos y formación de aminoácidos vinculados al potencial de asociación a superficies y sustratos para su crecimiento. L. plantarum tiene la particularidad de presentar capacidad antibacteriana frente a diferentes bacterias patógenas. El objetivo de este trabajo fue generar un modelo experimental de colonización por Lactobacillus plantarum LP5 en ratones (Mus musculus) destinado a mejorar el estado general del animal y prevenir la potencial presencia de patógenos zoonóticos.

Los ratones utilizados fueron hembras de la cepa Balb/cCmedc, de 6 semanas de edad. Los animales fueron dispuestos en 2 grupos de 3 individuos: tratado (GT) y control (GC). El GT fue administrado dos veces por semana mediante gavage con $100 \mu$ de LP5 de origen porcino resistente a rifampicina en una concentración de $10^{10}$ UFC. El GC fue manipulado en iguales condiciones, pero el probiótico fue reemplazado por solución fisiológica al $0,85 \%$. Los ratones fueron alimentados con agua y alimento ad libitum a lo largo del experimento (3 semanas). Las condiciones de alojamiento fueron estándar: $22-24{ }^{\circ} \mathrm{C}, 55 \pm 15 \%$ de humedad, $12 \mathrm{~h}$ luz/12 ciclo de oscuridad. La colonización en ambos grupos fue evaluada por la presencia de signos clínicos y mediante un análisis microbiológico de la materia fecal. La toma de muestra fue realizada a intervalos semanales para cuantificar las poblaciones del LP5 suministrado (en medio MRS suplementado con vancomicina y rifampicina), Lactobacillus spp. (en medio MRS suplementado con vancomicina), Escherichia coli (en medio TBX), coliformes totales (en medio VRBG), Campylobacter (en medio mCCDA para Campylobacter) y levaduras (en medio YGC modificado). El análisis microbiológico se realizó mediante recuento en placa para todas las poblaciones anteriormente descriptas.

Inicialmente la microbiota murina carecía de BAL resistentes a rifampicina, levaduras y Campylobacter termotolerantes, valores que se mantuvieron a lo largo del experimento. La generación de mutante de LP5 resistente a rifampicina facilitó su enumeración y permitió diferenciarlo fácilmente de la microbiota indígena. Los resultados del muestreo en el GT a la semana mostraron una recuperación de LP5 por encima de los 5 logUFC/g, a la segunda 7 logUFC/g y a la tercera fue de $5 \operatorname{logUFC} / \mathrm{g}$. El GC, en tanto, mantuvo el recuento nulo a lo largo del experimento. La microbiota láctica en ambos grupos se conservó desde el inicio en un orden de 9 logUFC/g y los coliformes a $37^{\circ} \mathrm{C}$ y $44^{\circ} \mathrm{C}$ se mantuvieron en $5 \operatorname{logUFC/g}$.
Los resultados mostraron que no existió un desbalance entre los componentes microbianos; por lo tanto, es factible afirmar que el inóculo no alteró el equilibrio del ecosistema intestinal y, en particular, no ejerció efecto inhibitorio sobre los integrantes de la microbiota previamente establecida. Otros estudios en donde se evalúe el potencial de inhibición in vivo de LP5 frente a un patógeno transmitido por los alimentos en un modelo de infección/colonización murino resulta interesante llevar a cabo en el futuro.

\section{Bibliografía}

Campero LM, Gos ML, Moore DP, Regidor Cerrillo JR, Unzaga JM, Moré GA, Ortega Mora LM, Venturini MC. 2018. Microsatellite pattern analysis of Neospora caninum from a naturally infected goat fetus. Vet. Parasitol. 255: 58-60.

Rosmini, M.; Sequeira, G.; Guerrero Legarreta, I.; Martí, L.; Dalla Santina, R.; Frizzo, L. (2004). Producción de Prebióticos para animales de abasto: importancia del uso de la microbiota intestinal indígena. Revista mexicana de ingeniería química, $3(2)$.

Signorini, M.; Soto, L.; Zbrun, M.; Sequeira, G.; Rosmini, M.; Frizzo L. (2012). Impact of probiotic administration on the health and fecal microbiota of young calves: A meta-analysis of randomized controlled trials of lactic acid bacteria. Research in Veterinary Science, 93(1), 250-8.

Vélez Zea, J.M.; Gutiérrez Ramírez, L.A.; Montoya O.I. (2013). Probióticos: una alternativa de producción limpia y de remplazo a los antibióticos promotores de crecimiento en la alimentación animal. Producción + Limpia, 8(1), 135-146. 